\title{
Synthesis of oleanolic acid saponins mimicking components of Chinese folk medicine Di Wu
}

\author{
Xing Huang ${ }^{\mathrm{a}, \mathrm{b}}$, Shuihong Cheng ${ }^{\mathrm{a}, \mathrm{b}}$, Yuguo Du ${ }^{\mathrm{a}, \mathrm{b}, *}$, Feihong Bing ${ }^{\mathrm{c}}$ \\ ${ }^{a}$ Research Center for Eco-Environmental Sciences, Chinese Academy of Sciences, Beijing 100085, China \\ ${ }^{\mathrm{b}}$ College of Chemistry and Chemical Engineering, Graduate University of Chinese Academy of Sciences, Beijing 100049, China \\ ${ }^{\mathrm{c}}$ Hubei College of Traditional Chinese Medicine, Hubei, China
}

\section{A R T I C L E I N F O}

\section{Article history:}

Received 14 February 2009

Received in revised form 25 March 2009

Accepted 7 April 2009

Available online 14 April 2009

\section{Keywords:}

Saponin

Apoptosis

Natural product

Chinese folk medicine

Cytotoxicity

\begin{abstract}
A B S T R A C T
3,28-Di-O-rhamnosylated oleanolic acid saponins, mimicking components of Chinese folk medicine $\mathrm{Di}$ $\mathrm{Wu}$, have been designed and synthesized. One-pot glycosylation and 'inverse procedure' technologies have been applied thus significantly simplifying the preparation of desired saponins. The cytotoxic activity of compounds $3-O-[\alpha-\mathrm{L}$-rhamnopyranosyl- $(1 \rightarrow 2)-\beta$-D-xylopyranosyl]oleanolic acid $28-O-[\alpha-\mathrm{L}-$ rhamnopyranosyl-( $1 \rightarrow 4)-\beta$-D-glucopyranosyl-( $1 \rightarrow 6)$ - $\beta$-D-glucopyranosyl] ester $(3), 3-0$-[ $\alpha$-L-rhamnopyranosyl]oleanolic acid 28-O-[ $\alpha$-L-rhamnopyranosyl- $(1 \rightarrow 4)-\beta$-D-glucopyranosyl-( $1 \rightarrow 6)-\beta$-D-glucopyranosyl] ester (4), 3-O-[ $\alpha$-L-rhamnopyranosyl]oleanolic acid 28-O-[ $\alpha$-L-rhamnopyranosyl] ester (5), and 3-O-[ $\alpha$-L-rhamnopyranosyl]oleanolic acid 28-O-[6-O-( $\alpha$-L-rhamnopyranosyl)hexyl] ester $(\mathbf{6})$ was preliminarily evaluated against HL-60 human promyelocytic leukemia cells. The natural saponin $\mathbf{3}$ and designed saponin 4 exhibited comparable moderate cytotoxic activity under our testing conditions.
\end{abstract}

() 2009 Elsevier Ltd. All rights reserved.

\section{Introduction}

Saponins, notably steroid or triterpene plant glycosides, have been used worldwide in the treatments of diseases and health care practices by taking advantages of their generally non-ionic surfactant and membrane-disrupting properties. ${ }^{1-5}$ The diversity of structures, the challenges of isolation and purification, their wide abundance in the plant kingdom and their broad spectrum of biological and pharmacological activities have driven chemists to the research field of saponins. ${ }^{6}$ It is surprising that more than half of the triterpene saponins are glycosides of oleanolic acid or its derivatives, with one sugar chain attached through an ether linkage at C3 and another through an ester linkage at C-28. ${ }^{7}$ In our ongoing project of $\mathrm{Di} \mathrm{Wu}{ }^{8}$ a Chinese folk medicine from dry rhizome of Anemone flaccida Fr. Schmidt, we proved that the major components (Scheme 1 ) of Di Wu are 3-O-[ $\alpha$-L-rhamnopyranosyl$(1 \rightarrow 2)$ - $\beta$-D-glucopyranosyl]oleanolic acid 28-O-[ $\alpha$-L-rhamnopyranosyl-( $1 \rightarrow 4)$ - $\beta$-D-glucopyranosyl-( $1 \rightarrow 6)$ - $\beta$-D-glucopyranosyl] ester (1), 3-O-[ $\alpha$-L-rhamnopyranosyl- $(1 \rightarrow 2)-\alpha$-L-arabinopyranosyl] oleanolic acid $28-O$-[ $\alpha$-L-rhamnopyranosyl-( $1 \rightarrow 4)-\beta$-D-glucopyranosyl$(1 \rightarrow 6)-\beta$-D-glucopyranosyl] ester (2), and 3-O-[ $\alpha$-L-rhamnopyranosyl-( $1 \rightarrow 2)-\beta$-D-xylopyranosyl]oleanolic acid $28-0$-[ $\alpha$-L-rhamnopyranosyl-( $1 \rightarrow 4)$ - $\beta$-D-glucopyranosyl-( $1 \rightarrow 6)-\beta$-D-glucopyranosyl] ester (3). ${ }^{9-11}$

\footnotetext{
* Corresponding author. Tel.: +86 10 62849126; fax: +86 1062923563 .

E-mail address: duyuguo@rcees.ac.cn (Y. Du).
}

Literature searching suggested that $\alpha$-L-rhamnopyranosyl moiety of some natural soponins may play a crucial role in triggering cell death by apoptosis. ${ }^{12}$ Several hypotheses have been proposed for the mechanism of their activity. ${ }^{13-15}$ However, the related SAR study is sporadic and unsystematic due to the difficulty in obtaining adequate analogical glycosides from nature. We have recently synthesized compound $\mathbf{3}$ which inhibited ConA-induced lymphocyte proliferation. ${ }^{8}$ However, the structural complexity, especially having $2^{\prime}-\mathrm{OH}$ branched sugar chain on $\mathrm{C}-3$ of oleanolic acid, has hindered the large-scale preparation of this type of bidesmosidic triterpene saponins. ${ }^{6,16-23}$ To simplify the structure and investigate the role of L-rhamnopyranosyl residues in oleananetype saponins, especially in Di Wu saponins, we here report the design, synthesis, and bioactivity of some structural analogues.

\section{Results and discussion}

The target molecules are shown in Scheme 2. To prepare compound 4, trisaccharide $\mathbf{1 0}$ was first synthesized via one-pot glycosylation technology (Scheme 3). Thus, 2,3,4-tri-O-benzoyl- $\alpha$ L-rhamnopyranosyl trichloroacetimidate $(\mathbf{8}, 1.4 \text { equiv })^{24}$ was slowly added into ethyl 2,3-di-O-acetyl-6-O-benzoyl-1-thio- $\beta$-Dglucopyranoside ${ }^{25}$ (7, 1.2 equiv) in dry $\mathrm{CH}_{2} \mathrm{Cl}_{2}$ in the presence of trimethylsilyl trifluoromethanesulfonate (TMSOTf, 0.17 equiv) at $-70^{\circ} \mathrm{C}$. The reaction mixture was warmed up to $\mathrm{rt}$ and stirred at this condition for another $30 \mathrm{~min}$, then acceptor 4-methoxyphenyl $2,3,4$-tri- $O$-acetyl- $\beta$-D-glucopyranoside $(\mathbf{9}, 1.0 \text { equiv })^{8,26}$ and 


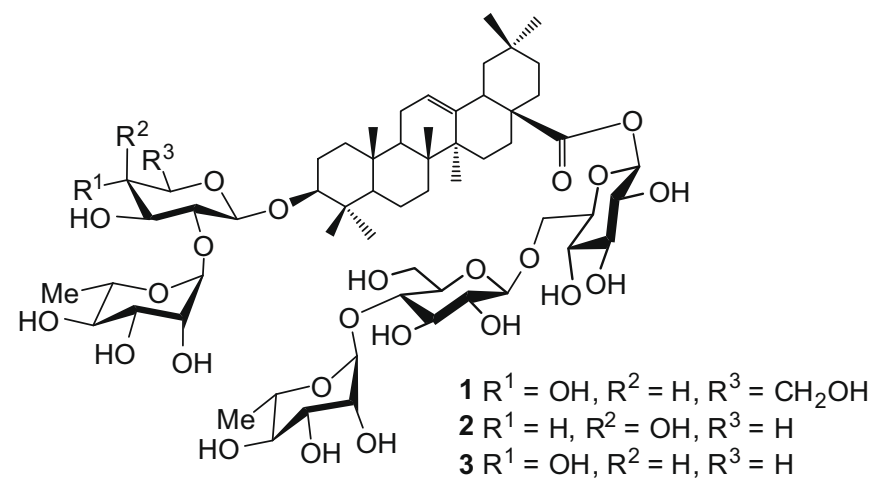

Scheme 1. Natural saponins isolated from Di Wu.
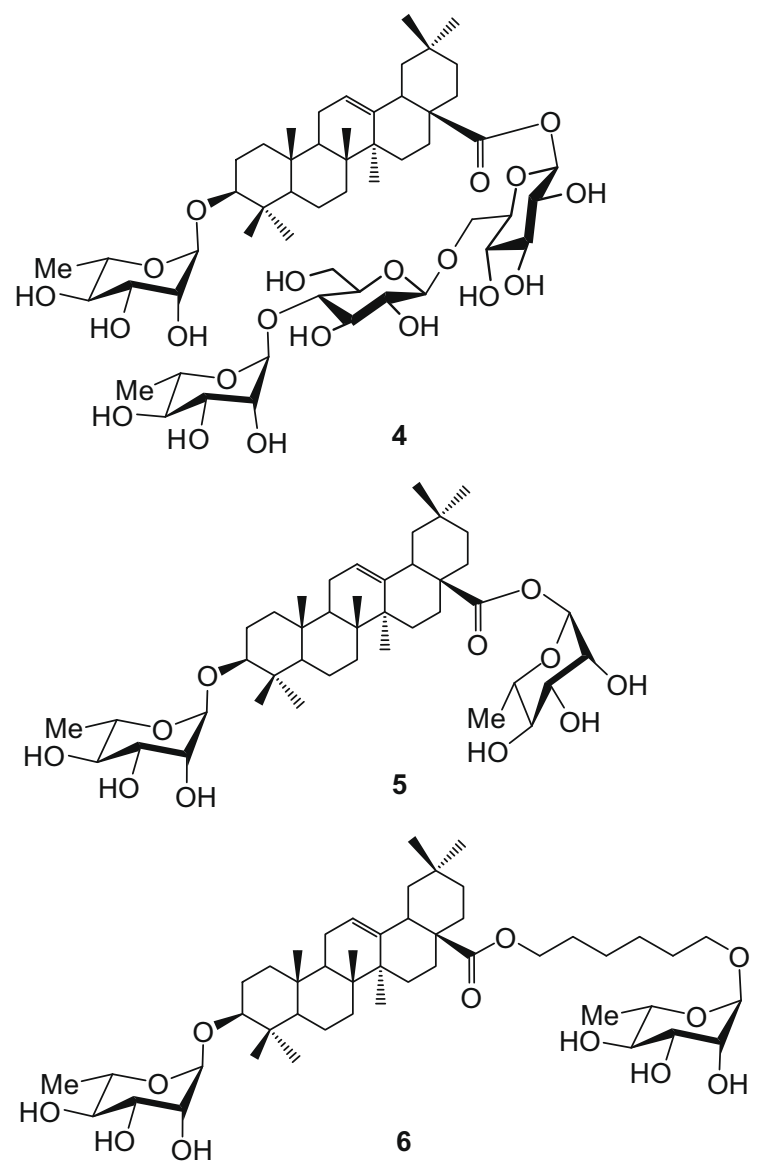

Scheme 2. Designed saponins mimicking natural Di Wu saponins.

$\mathrm{N}$-iodosuccinimide (NIS) in dry $\mathrm{CH}_{2} \mathrm{Cl}_{2}$, respectively, were added at $-15^{\circ} \mathrm{C}$, obtaining 4-methoxyphenyl 2,3,4-tri-O-benzoyl- $\alpha$-Lrhamnopyranosyl-( $1 \rightarrow 4)$-2,3-di-O-acetyl-6-O-benzoyl- $\beta$-D-glucopyranosyl-( $1 \rightarrow 6)-2,3,4$-tri-O-acetyl- $\beta$-D-glucopyranoside $(\mathbf{1 0})$ in a total yield of $53 \%$. Treatment of $\mathbf{1 0}$ with ceric ammonium nitrate (CAN) in $\mathrm{CH}_{3} \mathrm{CN}-\mathrm{H}_{2} \mathrm{O}(4: 1, \mathrm{v} / \mathrm{v})$, followed by trichloroacetimidation $\left(\mathrm{Cl}_{3} \mathrm{CCN}\right.$, DBU, $\left.\mathrm{CH}_{2} \mathrm{Cl}_{2}\right)$, ${ }^{27}$ furnished the trisaccharide donor 11 in $80 \%$ yield. Coupling of oleanolic trityl ester 12 with donor $\mathbf{8}$ was smoothly completed within $30 \mathrm{~min}$ in the presence of a catalytic amount of TMSOTf at $-35^{\circ} \mathrm{C}$, obtaining key intermediate $\mathbf{1 3}$ in 92\% yield (Scheme 4). Sugar-ester formation between trityl-ester $\mathbf{1 3}$ and trisaccharide donor $\mathbf{1 1}$ was achieved using a so-called 'Inverse Procedure $^{28,29}$ in dry dichloromethane under the promotion of TMSOTf leading to the fully protected saponin derivative 14 in excellent yield. Selective removal of the acetate and benzoate protecting groups in $\mathbf{1 4}$ with catalytic amount of $\mathrm{NaOMe}\left(\mathrm{CH}_{2} \mathrm{Cl}_{2}-\right.$ $\mathrm{MeOH}, 2: 1, \mathrm{v} / \mathrm{v}$ ), taking advantage of a kinetic steric effect of the neighboring quaternary center on $\mathrm{C}-28,{ }^{16}$ furnished the target 30 - $\alpha$-L-rhamnopyranosyl oleanolic acid $28-0-\alpha$-L-rhamnopyranosyl-( $1 \rightarrow 4)-\beta$-D-glucopyranosyl-( $1 \rightarrow 6)$ - $\beta$-D-glucopyranoside $(4)$ in 70\% yield from 12 .

Similarly (Scheme 5), condensation of $\mathbf{1 3}$ with $\mathbf{8}$ as described in the preparation of compound 14 gave derivative 15, which was subjected to deacylation with $\mathrm{NaOMe}$ in $\mathrm{CH}_{2} \mathrm{Cl}_{2}-\mathrm{MeOH}$ to give desired saponin 3-O- $\alpha$-L-rhamnopyranosyl oleanolic acid $28-0-\alpha-\mathrm{L}-$ rhamnopyranoside (5) in excellent overall yield.

In the preparation of compound 6 (Scheme 6), 3-O-acetyl oleanolic acid $\mathbf{1 6}^{30}$ was treated with oxalyl chloride in dry $\mathrm{CH}_{2} \mathrm{Cl}_{2}$, the resulting acid chloride was then immediately reacted with 1,6-hexanediol in the presence of triethylamine affording 3-Oacetyl oleanolic acid 28-O-(6-hydroxyhexyl) ester (17). Removal of acetyl group with $\mathrm{NaOMe}$ in $\mathrm{CH}_{2} \mathrm{Cl}_{2} / \mathrm{MeOH}(\rightarrow \mathbf{1 8})$, followed by glycosylation with $\mathbf{8}$ using the same procedure applied in the synthesis of 13, gave diglycosylated compound 19 in $90 \%$ yield. Global debenzoylation of 19 with catalytic amount of $\mathrm{NaOMe}\left(\mathrm{CH}_{2} \mathrm{Cl}_{2}-\right.$ $\mathrm{MeOH}, 2: 1, \mathrm{v} / \mathrm{v}$ ) in the presence of the $\mathrm{C}-28$ ester glycosidic linkage gave the target compound $\mathbf{6}$ in $60 \%$ yield from $\mathbf{1 6 .}$

The cytotoxic activities of compounds 3-6, and oleanolic acid were evaluated ${ }^{31,32}$ against HL-60 human promyelocytic leukemia cells (Table 1). Saponins $\mathbf{3}$ and $\mathbf{4}$ exhibited comparable moderate cytotoxic activity with $\mathrm{IC}_{50}$ values at 2.7 and $3.0 \mu \mathrm{g} / \mathrm{mL}$, respectively. Compared to 4, structural analogues $\mathbf{5}$ and $\mathbf{6}$ showed only weak activities under the same testing conditions, suggesting that sugar moieties (or may be polyhydroxyl linkers) are important between $\alpha$-L-rhamnopyranosyl residue and C-28 carboxyl group. Oleanolic acid itself was not cytotoxic under our assay conditions. The current results should be valuable for the related molecule design, synthesis, and bioactivity screening. ${ }^{33,34}$

\section{Experimental}

\subsection{General methods}

Optical rotations were determined at $25^{\circ} \mathrm{C}$ with a Perkin-Elmer Model 241-Mc automatic polarimeter. ${ }^{1} \mathrm{H}$ NMR, ${ }^{13} \mathrm{C}$ NMR, and ${ }^{1} \mathrm{H}-{ }^{1} \mathrm{H},{ }^{1} \mathrm{H}-{ }^{13} \mathrm{C}$ COSY spectra were recorded with a Bruker ARX 400 spectrometer for solutions in $\mathrm{CDCl}_{3}, \mathrm{MeOD}$, or DMSO- $d_{6}$. Chemical shifts are given in ppm downfield from internal $\mathrm{Me}_{4} \mathrm{Si}$. Mass spectra were measured using a MALDITOF-MS with $\alpha$-cyano-4-hydroxycinnamic acid as matrix. Thinlayer chromatography (TLC) was performed on silica gel $\mathrm{HF}_{254}$ with detection by charring with $30 \%(\mathrm{v} / \mathrm{v}) \mathrm{H}_{2} \mathrm{SO}_{4}$ in $\mathrm{MeOH}$, or by UV detector. Column chromatography was conducted by elution of a column of silica gel (100 mesh) with EtOAc-petroleum ether $\left(60-90^{\circ} \mathrm{C}\right)$ as the eluent, or a column of Biogel P2 with water as the eluent. Solutions were concentrated at $<50{ }^{\circ} \mathrm{C}$ under reduced pressure.

\subsection{4-Methoxyphenyl 2,3,4-tri-O -benzoyl- $\alpha$-L-rhamnopyrano- syl-( $1 \rightarrow 4)$-2,3-di-O-acetyl-6-O -benzoyl- $\beta$-D-glucopyranosyl- $(1 \rightarrow$ 6)-2,3,4-tri- $O$-acetyl- $\beta$-D-glucopyranoside (10)}

A suspension of 7 (495 mg, $1.2 \mathrm{mmol}), 8$ ( $870 \mathrm{mg}, 1.4 \mathrm{mmol}$ ), and $4 \AA \mathrm{MS}$ in dry $\mathrm{CH}_{2} \mathrm{Cl}_{2}(15 \mathrm{~mL})$ was stirred at $\mathrm{rt}$ for $0.5 \mathrm{~h}$, then cooled to $-70{ }^{\circ} \mathrm{C}$, and a solution of TMSOTf ( $\left.30 \mu \mathrm{L}, 0.17 \mathrm{mmol}\right)$ in $\mathrm{CH}_{2} \mathrm{Cl}_{2}(0.2 \mathrm{~mL})$ was added under $\mathrm{N}_{2}$ protection. The reaction mixture was allowed to stir at these conditions for about $3 \mathrm{~h}$, at the end of which time, TLC (petroleum ether-EtOAc 3:1) indicated a complete consumption of $\mathbf{8}$. To the above mixture was added 


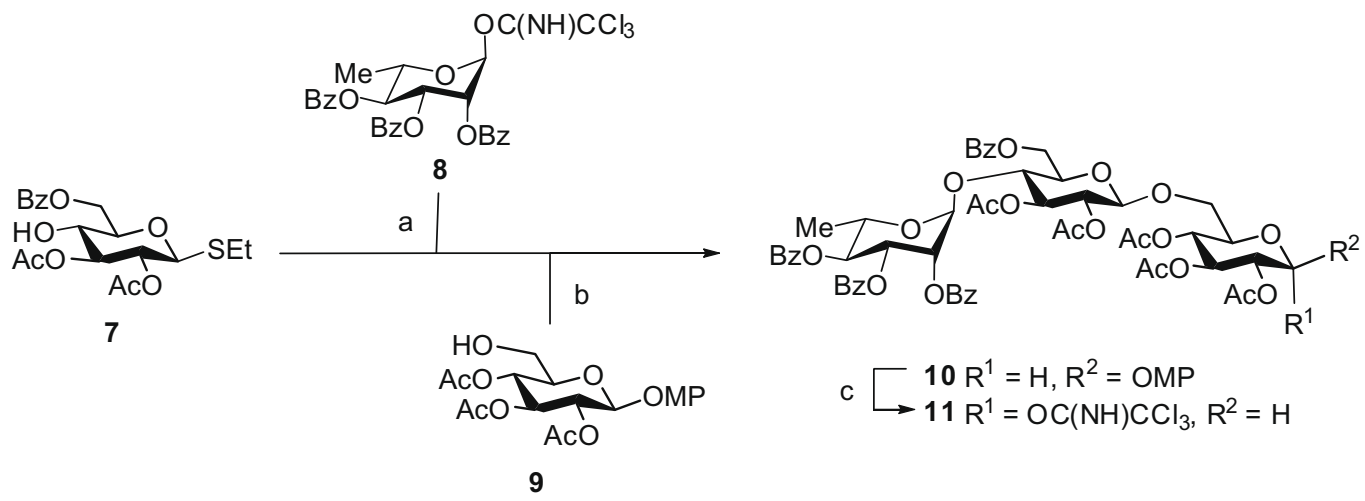

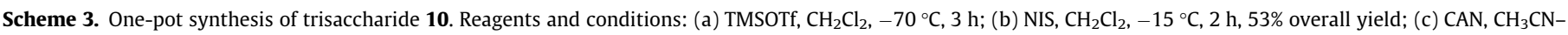
$\mathrm{H}_{2} \mathrm{O}(4: 1, \mathrm{v} / \mathrm{v})$; then $\mathrm{Cl}_{3} \mathrm{CCN}, \mathrm{DBU}, \mathrm{CH}_{2} \mathrm{Cl}_{2}, \mathrm{rt}, 80 \%$.

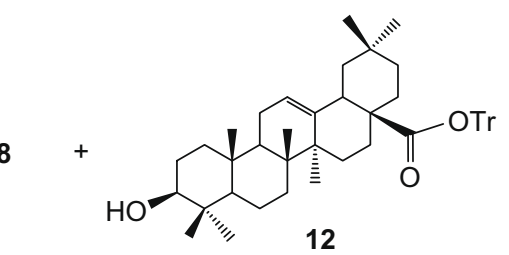

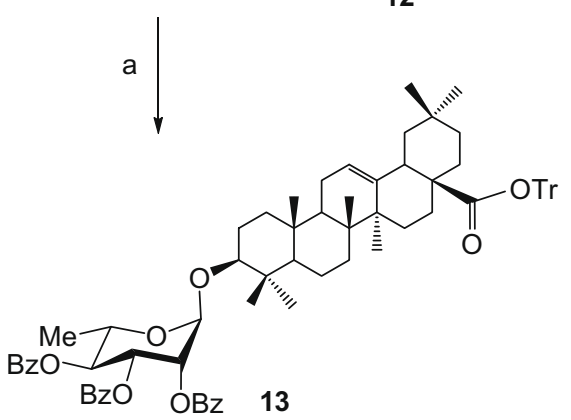

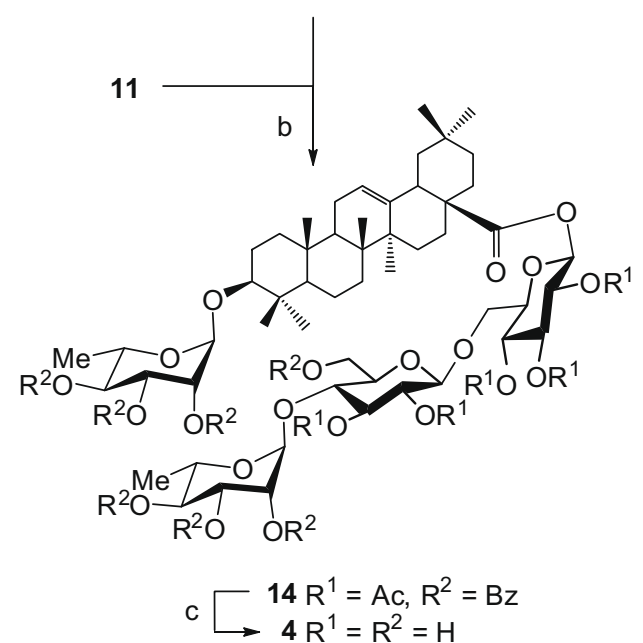

Scheme 4. Synthesis of saponin 4. Reagents and conditions: (a) TMSOTf, $\mathrm{CH}_{2} \mathrm{Cl}_{2}$ $-35^{\circ} \mathrm{C}, 92 \%$; (b) TMSOTf, $\mathrm{CH}_{2} \mathrm{Cl}_{2}, 0{ }^{\circ} \mathrm{C}, 1 \mathrm{~h}$, then $11,-35^{\circ} \mathrm{C}, 30 \mathrm{~min}, 90 \%$; (c) NaOMe, $\mathrm{CH}_{2} \mathrm{Cl}_{2}-\mathrm{MeOH}, 2: 1, \mathrm{v} / \mathrm{v}, 85 \%$.

compound 9 (412 $\mathrm{mg}, 1.0 \mathrm{mmol}$ ) in dry $\mathrm{CH}_{2} \mathrm{Cl}_{2}(2.5 \mathrm{~mL})$ at $-15^{\circ} \mathrm{C}$, followed by adding NIS ( $450 \mathrm{mg}, 2.0 \mathrm{mmol}$ ), and a further portion of TMSOTf $(10 \mu \mathrm{L}, 0.055 \mathrm{mmol})$ in $\mathrm{CH}_{2} \mathrm{Cl}_{2}$. The reaction mixture was stirred at these conditions for $2 \mathrm{~h}$, then quenched by $\mathrm{Et}_{3} \mathrm{~N}$, diluted with $\mathrm{CH}_{2} \mathrm{Cl}_{2}$, washed with satd aq $\mathrm{NaHCO}_{3}$ and water. The organic layer was combined, dried, and concentrated. Purification by column chromatography (1:1 petroleum ether-EtOAc) gave trisac- charide 10 as a foamy solid (640 mg, 53\% overall yield), which presented the same physical data as that reported before $\left\{[\alpha]_{D}^{25}+75\right.$ (c 2, $\mathrm{CHCl}_{3}$ ), lit. ${ }^{8}[\alpha]_{\mathrm{D}}^{25}+74$ (c 2.1, $\left.\left.\mathrm{CHCl}_{3}\right)\right\}$.

\subsection{3-0-[2,3,4-Tri-O-benzoyl- $\alpha$-L-rhamnopyranosyl]oleanolic acid 28-O-trityl ester (13)}

To a mixture of compounds $8(220 \mathrm{mg}, 0.35 \mathrm{mmol})$ and $\mathbf{1 2}$ (200 $\mathrm{mg}, 0.29 \mathrm{mmol}$ ) in anhyd $\mathrm{CH}_{2} \mathrm{Cl}_{2}(5 \mathrm{~mL})$ was added TMSOTf ( $7 \mu \mathrm{L}, 0.04 \mathrm{mmol}$ ) under an $\mathrm{N}_{2}$ atmosphere at $-35^{\circ} \mathrm{C}$. The mixture was stirred under these conditions for $30 \mathrm{~min}$, at the end of which time TLC (3:1 petroleum ether-EtOAc) indicated that all starting materials were consumed. The reaction mixture was neutralized with $\mathrm{Et}_{3} \mathrm{~N}$ and concentrated. Column chromatography (4:1 petroleum ether-EtOAc) of the residue gave $\mathbf{1 3}$ as a foamy solid (305 mg, 92\%): $[\alpha]_{\mathrm{D}}^{25}+45\left(\right.$ c 1, $\left.\mathrm{CHCl}_{3}\right) ;{ }^{1} \mathrm{H}$ NMR $\left(400 \mathrm{MHz}, \mathrm{CDCl}_{3}\right)$ : $\delta 0.35,0.82,0.88,0.90,0.91,1.11,1.29,1.31\left(8 \mathrm{~s}, 8 \times 3 \mathrm{H}, 8 \mathrm{CH}_{3}\right)$, 0.78-1.79 (m, 22H), 2.83 (dd, $1 \mathrm{H}, J 4.0,13.3 \mathrm{~Hz}, \mathrm{H}-18$ of oleanolic acid), 3.25 (dd, $1 \mathrm{H}, J 4.6,11.5 \mathrm{~Hz}, \mathrm{H}-3$ of oleanolic acid), $4.28-$ 4.31 (m, 1H, H-5), 5.07 (d, 1H, J 2.0 Hz, H-1), 5.24 (br s, 1H, H-12 of oleanolic acid), 5.62-5.69 (m, 2H, H-2, H-4), 5.82 (dd, $1 \mathrm{H}, J 3.2$, $10.1 \mathrm{~Hz}, \mathrm{H}-3), 7.20-8.09(\mathrm{~m}, 30 \mathrm{H}, 6 \mathrm{Ph})$. Anal. Calcd for $\mathrm{C}_{76} \mathrm{H}_{84} \mathrm{O}_{10}$ : C, 78.86; H, 7.31; Found: C, 79.14; H, 7.22.

\subsection{3-O-[2,3,4-Tri- $O$-benzoyl- $\alpha$-L-rhamnopyranosyl]oleanolic acid 28-0-[2,3,4-tri-O-benzoyl- $\alpha$-L-rhamnopyranosyl- $(1 \rightarrow 4)$ - 2,3-di-O-acetyl-6-O-benzoyl- $\beta$-D-glucopyranosyl- $(1 \rightarrow 6)-2,3,4-$ tri-0-acetyl- $\beta$-D-glucopyranosyl] ester (14)}

To a solution of compound $13(243 \mathrm{mg}, 0.21 \mathrm{mmol})$ in dry dichloromethane $(5 \mathrm{~mL})$ was added TMSOTf $(20 \mu \mathrm{L}, 0.11 \mathrm{mmol})$ under an $\mathrm{N}_{2}$ atmosphere at $0^{\circ} \mathrm{C}$. The mixture was stirred at this condition for $1 \mathrm{~h}$, then cooled to $-35^{\circ} \mathrm{C}$, and trisaccharide $\mathbf{1 1}$ (306 mg, $0.24 \mathrm{mmol}$ ) in anhyd $\mathrm{CH}_{2} \mathrm{Cl}_{2}(3 \mathrm{~mL}$ ) was added dropwise. The reaction mixture was stirred under these conditions for further $30 \mathrm{~min}$, quenched by $\mathrm{Et}_{3} \mathrm{~N}$, and concentrated. The residue was purified by silica gel column chromatography (7:2, petroleum etherEtOAc) to give compound $\mathbf{1 4}$ as a foamy solid (381 $\mathrm{mg}, 90 \%$ ): $[\alpha]_{\mathrm{D}}^{25}+98\left(\right.$ c $\left.1.1, \mathrm{CHCl}_{3}\right) ;{ }^{1} \mathrm{H}$ NMR $\left(400 \mathrm{MHz}, \mathrm{CDCl}_{3}\right): 0.76,0.85$, $0.89,0.95,0.99,1.12,1.17,1.29,1.56\left(9 \mathrm{~s}, 9 \times 3 \mathrm{H}, 9 \mathrm{CH}_{3}\right), 0.76-$ $1.79(\mathrm{~m}, 22 \mathrm{H}), 1.99,2.00,2.02,2.08,2.11(5 \mathrm{~s}, 5 \times 3 \mathrm{H}, 5 \mathrm{Ac}), 2.80$ (dd, $1 \mathrm{H}, J 4.2,10.1 \mathrm{~Hz}, \mathrm{H}-18$ of oleanolic acid), 3.22 (dd, $1 \mathrm{H}, J$ 7.3, $11.6 \mathrm{~Hz}, \mathrm{H}-3$ of oleanolic acid), 3.60 (dd, $1 \mathrm{H}, J 5.6,11.5 \mathrm{~Hz}, \mathrm{H}-6 \mathrm{a}^{\mathrm{Glc}}$ ), $3.79-3.89(\mathrm{~m}, 3 \mathrm{H}), 4.05\left(\mathrm{t}, 1 \mathrm{H}, J 9.3 \mathrm{~Hz}, \mathrm{H}-5^{\mathrm{Glc}}\right), 4.14-4.16(\mathrm{~m}, 1 \mathrm{H}$, $\left.\mathrm{H}-5^{\mathrm{Glc}}\right), 4.30-4.33\left(\mathrm{~m}, 1 \mathrm{H}, \mathrm{H}-5^{\mathrm{Rha}}\right), 4.60-4.64(\mathrm{~m}, 2 \mathrm{H}), 4.86-4.99$ (m, 3H), 5.07 (d, 1H, J $\left.2.1 \mathrm{~Hz}, \mathrm{H}-1^{\text {Rha }}\right), 5.13-5.33(\mathrm{~m}, 5 \mathrm{H}), 5.51$ (br $\mathrm{s}, 1 \mathrm{H}, \mathrm{H}-12$ of oleanolic acid), 5.55-5.80 (m, 6H), 7.23-8.09 (m, $35 \mathrm{H}, 7 \mathrm{Ph}) .{ }^{13} \mathrm{C}$ NMR $\left(100 \mathrm{MHz}, \mathrm{CDCl}_{3}\right): 175.9,170.8,170.7,170.5$, 
13

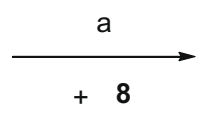

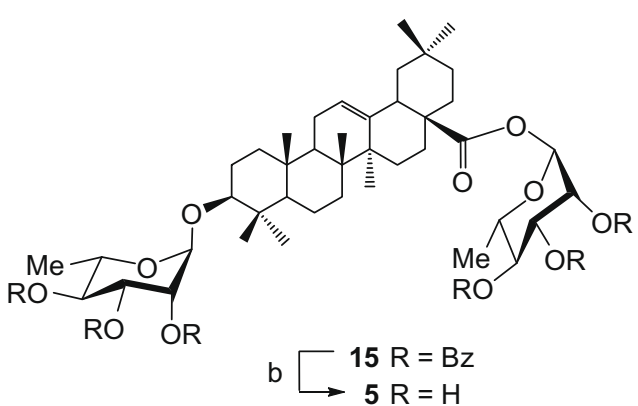

Scheme 5. Synthesis of saponin 5. Reagents and conditions: (a) TMSOTf, $\mathrm{CH}_{2} \mathrm{Cl}_{2}, 0{ }^{\circ} \mathrm{C}, 1 \mathrm{~h}$, then $8,-35{ }^{\circ} \mathrm{C}, 30 \mathrm{~min}, 93 \%$; $(\mathrm{b}) \mathrm{NaOMe}, \mathrm{CH} \mathrm{Cl}_{2}-\mathrm{MeOH} 2: 1, \mathrm{v} / \mathrm{v}, 92 \%$.

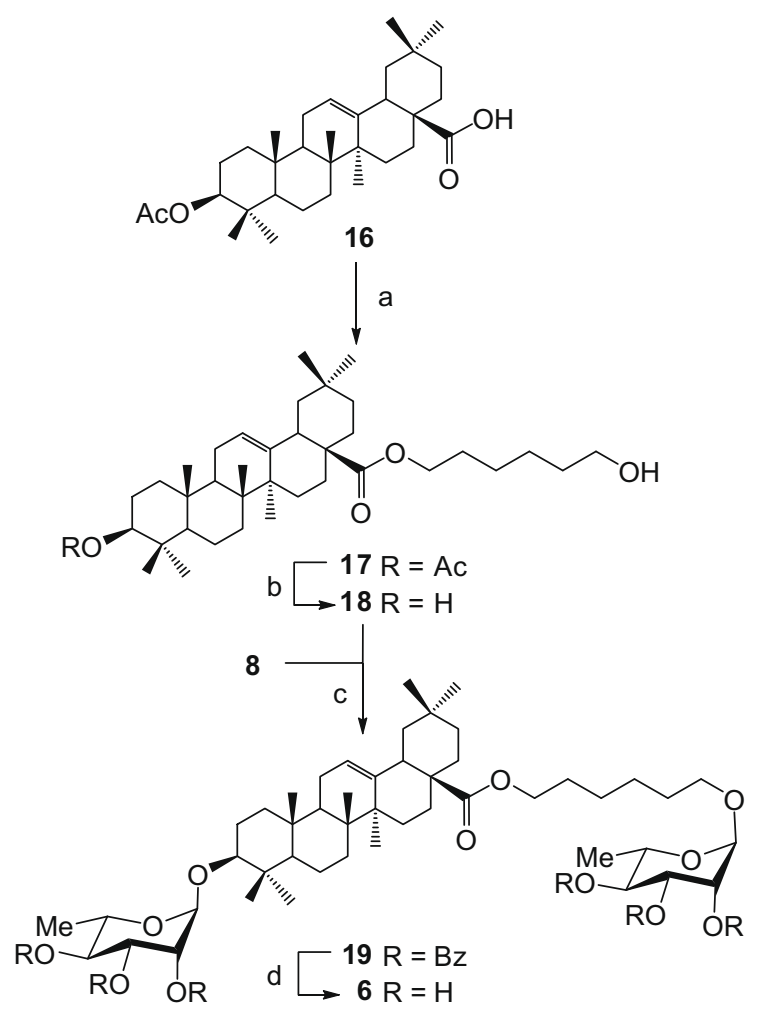

Scheme 6. Synthesis of saponin 6. Reagents and conditions: (a) oxalyl chloride, $\mathrm{CH}_{2} \mathrm{Cl}_{2}$; 1,6-hexanediol, triethylamine, 82\%; (b) NaOMe, MeOH, 91\%; (c) TMSOTf, $\mathrm{CH}_{2} \mathrm{Cl}_{2},-35^{\circ} \mathrm{C}, 90 \%$; (d) NaOMe, $\mathrm{CH}_{2} \mathrm{Cl}_{2}-\mathrm{MeOH}, 2: 1, \mathrm{v} / \mathrm{v}, 89 \%$.

Table 1

Cytotoxicity of compounds 3-6 and oleanolic acid against HL-60 Cells ${ }^{\mathrm{a}}$

\begin{tabular}{lll}
\hline Entry & Compound & $\mathrm{IC}_{50}(\mu \mathrm{g} / \mathrm{mL})$ \\
\hline 1 & $\mathbf{3}$ & 2.7 \\
2 & $\mathbf{4}$ & 3.0 \\
3 & $\mathbf{5}$ & 20.1 \\
4 & $\mathbf{6}$ & 14.5 \\
5 & Oleanolic acid & $>30$ \\
\hline
\end{tabular}

${ }^{a}$ The cells were continuously treated with each sample for $72 \mathrm{~h}$, and the cell growth was evaluated using MTT reduction assay. Data are mean values of three experiments performed in triplicate.

170.0, 169.5, 166.5, 166.3, 166.3, 166.2, 166.0, 166.0, 142.0, 134.0, $134.1,133.8,133.7,133.6,130.5,130.3,130.3,130.1,130.0,129.9$, $129.8,129.8,129.7,129.1,129.0,129.0,128.8,124.0,100.1$ (C-1), 99.8 (C-1), 99.7 (C-1), 92.1 (C-1), 89.5, 74.8, 74.5, 73.6, 73.5, 72.6, $72.5,72.0,71.8,71.7,70.8,70.6,70.0,69.4,68.7,68.2,67.4,56.0$, 54.4, 48.2, 47.3, 46.4, 42.4, 41.7, 39.9, 39.6, 39.0, 37.4, 34.3, 33.5,
32.3, 31.1, 29.9, 28.9, 28.4, 26.2, 26.0, 24.1, 23.5, 21.7, 21.3, 21.2, 18.9, 18.1, 18.0, 17.6, 17.2, 16.0. Anal. Calcd for $\mathrm{C}_{113} \mathrm{H}_{126} \mathrm{O}_{33}$ : C, 67.45; H, 6.31; Found: C, 67.21; H, 6.27.

\subsection{3-O-[ $\alpha$-L-Rhamnopyranosyl]oleanolic acid 28-0-[ $\alpha-\mathrm{L}-$ rhamnopyranosyl- $(1 \rightarrow 4)-\beta$-D-glucopyranosyl- $(1 \rightarrow 6)-\beta-D-$ glucopyranosyl] ester (4)}

To a solution of $14(202 \mathrm{mg}, 0.1 \mathrm{mmol})$ in dry $\mathrm{CH}_{2} \mathrm{Cl}_{2}-\mathrm{MeOH}$ $(1: 2, \mathrm{v} / \mathrm{v}, 15 \mathrm{~mL})$ was added $1.0 \mathrm{M} \mathrm{NaOMe}$ in $\mathrm{MeOH}$ at $0{ }^{\circ} \mathrm{C}$. The mixture was stirred at $\mathrm{rt}$ until all starting materials were consumed, and then neutralized with Dowex $\mathrm{H}^{+}$resin. The mixture was filtered and filtrate was concentrated under diminished pressure. The resulting residue was purified by Biogel P2 column chromatography using $\mathrm{H}_{2} \mathrm{O}$ as eluent to afford, after freeze drying, compound 4 as an amorphous solid (91 mg, 85\%): $[\alpha]_{\mathrm{D}}^{25}+7.8$ (c 1, $\mathrm{MeOH}) ;{ }^{1} \mathrm{H}$ NMR (400 MHz, MeOD): $0.81\left(\mathrm{~s}, 6 \mathrm{H}, 2 \mathrm{CH}_{3}\right), 0.92-0.97$ $\left(\mathrm{m}, 12 \mathrm{H}, 4 \mathrm{CH}_{3}\right), 1.17\left(\mathrm{~s}, 3 \mathrm{H}, \mathrm{CH}_{3}\right), 1.24(\mathrm{~d}, 3 \mathrm{H}, J 6.2 \mathrm{~Hz}), 1.27$ (d, $3 \mathrm{H}, J 6.4 \mathrm{~Hz}$ ), $0.81-1.71 \mathrm{~m}, 22 \mathrm{H}), 2.86$ (dd, $1 \mathrm{H}, \mathrm{H}-18$ of oleanolic acid), 3.09 (dd, $1 \mathrm{H}, J 6.9,11.1 \mathrm{~Hz}, \mathrm{H}-3$ of oleanolic acid), 3.23$3.84(\mathrm{~m}, 19 \mathrm{H}), 3.96-3.98(\mathrm{~m}, 1 \mathrm{H}), 4.10(\mathrm{~d}, 1 \mathrm{H}, \mathrm{J} 10.8 \mathrm{~Hz}), 4.74(\mathrm{~d}$, $1 \mathrm{H}, J 7.8 \mathrm{~Hz}), 4.41$ (d, $1 \mathrm{H}, J 7.8 \mathrm{~Hz}$ ), 5.27 (br s, $1 \mathrm{H}, \mathrm{H}-12$ of oleanolic acid), 5.35 (d, $1 \mathrm{H}, J 8.0 \mathrm{~Hz}) .{ }^{13} \mathrm{C}$ NMR (100 MHz, MeOD): 171.2, 144.1, 122.4, 103.0 (C-1), 102.9 (C-1), 101.5 (C-1), 94.3 (C-1), 89.0, 78.7, 77.4, 77.2, 76.0, 75.9, 74.5, 73.3, 73.0, 72.9, 71.8, 71.7, 71.6, 71.4, 79.1, 69.8, 69.1, 68.6, 61.2, 55.4, 46.4, 42.1, 41.7, 39.9, $39.2,38.9,37.1,34.1,33.1,32.8,32.4,30.8,28.0,25.6,23.4,18.7$, 17.1, 17.1, 17.0, 16.3, 15.3. MALDITOF-MS: calcd for $\mathrm{C}_{54} \mathrm{H}_{88} \mathrm{O}_{21}$ : $1072.58[\mathrm{M}]^{+}$; found, $1095.6[\mathrm{M}+\mathrm{Na}]^{+}$. Anal. Calcd for $\mathrm{C}_{54} \mathrm{H}_{88} \mathrm{O}_{21}$ : C, 60.43; H, 8.26; Found: C, 60.67; H, 8.32.

\subsection{3-O-[2,3,4-Tri-O -benzoyl- $\alpha$-L-Rhamnopyranosyl]oleanolic} acid 28-0-[2,3,4-tri-O-benzoyl- $\alpha$-L-rhamnopyranosyl] ester (15)

Coupling of compounds 8 (248 mg, $0.4 \mathrm{mmol}$ ) and 13 (417 mg, $0.36 \mathrm{mmol}$ ) using the same procedure as described in the preparation of 14 from 11 and 13 obtained compound 15 as a foamy solid (460 mg, 93\%): $\left[\alpha_{\mathrm{D}}^{25}+43\right.$ (c 1, $\left.\mathrm{CHCl}_{3}\right) ;{ }^{1} \mathrm{H}$ NMR (400 MHz, $\mathrm{CDCl}_{3}$ ): $0.79,0.87,0.88,0.90,1.00,1.01,1.14(7 \mathrm{~s}, 7 \times 3 \mathrm{H}), 1.26(\mathrm{~d}, 3 \mathrm{H}, J$ $6.2 \mathrm{~Hz}, \mathrm{H}-6)), 1.30$ (d, 3H, J $6.2 \mathrm{~Hz}, \mathrm{H}-6)), 0.73-1.86(\mathrm{~m}, 22 \mathrm{H})$, 2.97 (dd, $1 \mathrm{H}, J 3.6,13.4 \mathrm{~Hz}, \mathrm{H}-18$ of oleanolic acid), 3.17 (dd, $1 \mathrm{H}, J$ $6.9,10.8 \mathrm{~Hz}, \mathrm{H}-3$ of oleanolic acid), 4.19-4.23 (m, 2H, 2H-5), 5.02 (d, $1 \mathrm{H}, J 1.9 \mathrm{~Hz}, \mathrm{H}-1$ ), 5.36 (br s, 1H, H-12 of oleanolic acid), 5.57-5.79 (m, 6H), 6.22 (d, $1 \mathrm{H}, J 1.8 \mathrm{~Hz}, \mathrm{H}-1), 7.19-8.04(\mathrm{~m}, 30 \mathrm{H}$, 6Ph). ${ }^{13} \mathrm{C}$ NMR (100 MHz, $\left.\mathrm{CDCl}_{3}\right): 175.4,166.5,166.3,166.2$ (2C), $166.1,165.8,144.5,134.2,134.1,134.0,133.8$ (2C), 133.6, 131.5, 130.6, 130.5, 130.3, 130.2, 130.0, 129.9, 129.8 (2C), 129.7, 129.4, 129.2 (2C), 129.1, 129.0, 128.9, 128.8, 124.0, 100.3 (C-1), 90.9 (C1), 73.4, 72.6, 71.8, 71.1, 70.8, 70.5, 70.3, 69.8, 69.7, 67.4, 66.1, $48.1,48.0,46.1,42.6,42.5,40.1,39.6,39.1,37.4,34.3,33.7,33.4$, $33.2,31.4,31.2,30.3,29.0,28.2,26.5,26.0,24.1,23.7,21.6,19.8$, 
18.9, 18.4, 18.3, 18.2, 17.8, 17.2, 16.0, 14.3. Anal. Calcd for $\mathrm{C}_{84} \mathrm{H}_{92} \mathrm{O}_{17}$ : C, 73.45; H, 6.75; Found: C, 73.72; H, 6.68.

\subsection{3- $\Theta[\alpha-\mathrm{L}-\mathrm{Rh}$ amnopyranosyl]oleanolic acid 28-O-[ $\alpha$-L-rhamno- pyranosyl] ester (5)}

Compound 15 (215 mg, $0.16 \mathrm{mmol}$ ) was treated with NaOMe, as described in the preparation of $\mathbf{4}$, affording compound $\mathbf{5}$ (108 mg, 92\%) as a foamy solid: ${ }^{1} \mathrm{H}$ NMR (400 MHz, MeOD): $\left[\alpha_{\mathrm{D}}^{25}\right.$ $+11\left(c 1.5, \mathrm{H}_{2} \mathrm{O}\right) ; \delta$ 0.79-1.91 (m, 49H), 2.90 (dd, $1 \mathrm{H}, \mathrm{H}-18$ of oleanolic acid), 3.09 (dd, 1H, H-3 of oleanolic acid), 3.35 (t, 1H, J $9.5 \mathrm{~Hz}$ ), $3.43(\mathrm{t}, 1 \mathrm{H}, J 9.5 \mathrm{~Hz}), 3.61-3.72(\mathrm{~m}, 4 \mathrm{H}), 3.75(\mathrm{br} \mathrm{s}, 1 \mathrm{H}), 3.82(\mathrm{br} \mathrm{s}$, $1 \mathrm{H}), 4.72(\mathrm{~d}, 1 \mathrm{H}, J 2.1 \mathrm{~Hz}, \mathrm{H}-1), 5.31$ (br s, $1 \mathrm{H}, \mathrm{H}-12$ of oleanolic acid), 5.92 (d, $1 \mathrm{H}, J 2.3 \mathrm{~Hz}, \mathrm{H}-1) .{ }^{13} \mathrm{C}$ NMR (100 MHz, DMSO- $\left.d_{6}\right)$ : 175.3, 143.6, 122.7, 103.1 (C-1), 93.6 (C-1), 87.8, 72.4, 71.8, 71.4, 71.1, 71.0, 70.9, 69.9, 68.8, 55.2, 47.3, 46.9, 45.7, 41.6, 41.5, 38.4, $36.7,33.5,33.1,32.8,32.5,30.8,28.3,27.35,25.98,26.0,25.3$, 23.7, 23.4, 22.8, 18.2, 18.1, 17.3, 16.8, 15.5. MALDITOF-MS: calcd for $\mathrm{C}_{42} \mathrm{H}_{68} \mathrm{O}_{11}$ : $748.5[\mathrm{M}]^{+}$; found, $771.88[\mathrm{M}+\mathrm{Na}]+$. Anal. Calcd for $\mathrm{C}_{42} \mathrm{H}_{68} \mathrm{O}_{11}$ : C, 67.35; H, 9.15; Found: C, 67.60; H, 9.08.

\subsection{3-0-Acetyl oleanolic acid 28-0-(6-hydroxyhexyl) ester (17)}

To a stirred solution of $\mathbf{1 6}(500 \mathrm{mg}, 1.0 \mathrm{mmol})$ and $N, N$-dimethylformamide ( $125 \mu \mathrm{L}, 1.50 \mathrm{mmol})$ in dry $\mathrm{CH}_{2} \mathrm{Cl}_{2}(5 \mathrm{~mL})$ was added oxalyl chloride $(430 \mu \mathrm{L}, 5.01 \mathrm{mmol})$ at room temperature under nitrogen atmosphere. The reaction mixture was stirred at this condition for $1 \mathrm{~h}$, then the organic layer was transferred into a solution of 1,6-hexanediol $(354 \mathrm{mg}, 3.00 \mathrm{mmol})$, triethylamine $(0.85 \mathrm{~mL}$, $6.00 \mathrm{mmol}$ ), and 4-methylaminopyridine $(15 \mathrm{mg})$ in dry $\mathrm{CH}_{2} \mathrm{Cl}_{2}$ $(20 \mathrm{~mL})$ at $\mathrm{rt}$ under $\mathrm{N}_{2}$ protection. The mixture was stirred for $6 \mathrm{~h}$, then poured into cold water, and the organic phase was washed with $\mathrm{NaHCO}_{3}$ aqueous solution, dried over $\mathrm{Na}_{2} \mathrm{SO}_{4}$, and concentrated. The crude product was purified by flash column chromatography (petroleum ether-EtOAc 4:1) to give $\mathbf{1 7}$ (491 mg, 82\%) as an amorphous solid: $\left[\alpha_{\mathrm{D}}^{25}+65\left(\right.\right.$ c $\left.0.5, \mathrm{CHCl}_{3}\right) ;{ }^{1} \mathrm{H}$ $\operatorname{NMR}\left(400 \mathrm{MHz}, \mathrm{CDCl}_{3}\right): 0.74,0.86,0.87,0.90,0.93,0.93,1.13$ (7s, $\left.7 \times 3 \mathrm{H}, 7 \mathrm{CH}_{3}\right), 0.74-1.87(\mathrm{~m}, 30 \mathrm{H}), 2.05(\mathrm{~s}, 3 \mathrm{H}, \mathrm{Ac}), 2.87(\mathrm{dd}, 1 \mathrm{H}$, $J 4.0,13.8 \mathrm{~Hz}, \mathrm{H}-18$ of oleanolic acid), 4.49 (t, $1 \mathrm{H}, J 8.1 \mathrm{~Hz}, \mathrm{H}-3$ of oleanolic acid), 3.63-3.67 (m, $\left.2 \mathrm{H}, \mathrm{CH}_{2} \mathrm{OH}\right), 3.99-4.03(\mathrm{~m}, 2 \mathrm{H}$, $\mathrm{CH}_{2} \mathrm{OC}=\mathrm{O}$ ), 5.28 (br s, $1 \mathrm{H}, \mathrm{H}-12$ of oleanolic acid). Anal. Calcd for $\mathrm{C}_{38} \mathrm{H}_{62} \mathrm{O}_{5}$ : C, 76.21; $\mathrm{H}, 10.43$; Found: C, 75.96; $\mathrm{H}, 10.40$.

\subsection{Oleanolic acid 28-0-(6-hydroxyhexyl) ester (18)}

$1 \mathrm{M}$ NaOMe was added into a solution of compound 17 (443 mg, $0.74 \mathrm{mmol})$ in $\mathrm{MeOH} / \mathrm{CH}_{2} \mathrm{Cl}_{2}(3 \mathrm{~mL} / 7 \mathrm{~mL})$. The reaction mixture was stirred under reflux for $72 \mathrm{~h}$ at $\mathrm{pH} 9.5$, then neutralized with Dowex-50 $\left(\mathrm{H}^{+}\right)$ion exchange resin. The mixture was filtered, the filtrate concentrated, and the resulting residue was purified by silica gel column chromatography (petroleum ether-EtOAc 2:1) to afford compound 18 as a white foam (375 mg, 91\%): $\left[\alpha_{D}^{25}+72\right.$ (c 1 , $\left.\mathrm{CHCl}_{3}\right) ;{ }^{1} \mathrm{H}$ NMR $\left(400 \mathrm{MHz}, \mathrm{CDCl}_{3}\right): 0.73,0.77,0.90,0.90,0.92$, $0.98,1.13\left(7 \mathrm{~s}, 7 \times 3 \mathrm{H}, 7 \mathrm{CH}_{3}\right), 0.73-2.01(\mathrm{~m}, 30 \mathrm{H}), 2.86(\mathrm{dd}, 1 \mathrm{H}, J$ $4.4,13.9 \mathrm{~Hz}, \mathrm{H}-18$ of oleanolic acid), 3.21 (dd, $1 \mathrm{H}, J 4.3,10.8 \mathrm{~Hz}$, $\mathrm{H}-3$ of oleanolic acid), 3.63-3.67 (m, $\left.2 \mathrm{H}, \mathrm{CH}_{2} \mathrm{OH}\right), 3.98-4.03(\mathrm{~m}$, $2 \mathrm{H}, \mathrm{CH}_{2} \mathrm{OC}=\mathrm{O}$ ), 5.27 (br s, $1 \mathrm{H}, \mathrm{H}-12$ of oleanolic acid). Anal. Calcd for $\mathrm{C}_{36} \mathrm{H}_{60} \mathrm{O}_{4}$ : C, 77.65; $\mathrm{H}, 10.86$; Found: $\mathrm{C}, 77.91 ; \mathrm{H}, 10.78$.

3.10. 3-O-[2,3,4-Tri-O-benzoyl- $\alpha$-L-Rhamnopyranosyl]oleanolic acid 28-0-[6-0 -(2,3,4-tri-O -benzoyl- $\alpha$-L-rhamnopyranosyl)hexyl] ester (19)

To a solution of compounds $8(480 \mathrm{mg}, 0.77 \mathrm{mmol})$ and 18 (200 $\mathrm{mg}, 0.36 \mathrm{mmol}$ ) in anhyd $\mathrm{CH}_{2} \mathrm{Cl}_{2}(5 \mathrm{~mL})$ was added TMSOTf $(16 \mu \mathrm{L}, 0.09 \mathrm{mmol})$ under an $\mathrm{N}_{2}$ atmosphere at $-35^{\circ} \mathrm{C}$. The reac- tion mixture was stirred under these conditions for $30 \mathrm{~min}$, at the end of which time TLC (petroleum ether-EtOAc 5:2) indicated that all starting materials were consumed. The reaction mixture was neutralized with $\mathrm{Et}_{3} \mathrm{~N}$ and concentrated. Column chromatography of the residue gave 19 as a white foam $(477 \mathrm{mg}, 90 \%)$ : $\left[\alpha_{D}^{25}\right.$ +89 (c 0.5, $\left.\mathrm{CHCl}_{3}\right) ;{ }^{1} \mathrm{H}$ NMR $\left(400 \mathrm{MHz}, \mathrm{CDCl}_{3}\right): 0.79,0.88,0.92$, $0.96,1.00,1.08,1.16\left(7 \mathrm{~s}, 7 \times 3 \mathrm{H}, 7 \mathrm{CH}_{3}\right), 1.34,1.39(2 \mathrm{~d}, 2 \times 3 \mathrm{H}, J$ $\left.6.3 \mathrm{~Hz}, 2 \mathrm{H}-6^{\text {Rha }}\right), 0.79-1.82(\mathrm{~m}, 3 \mathrm{OH}), 2.90(\mathrm{dd}, 1 \mathrm{H}, \mathrm{H}-18$ of oleanolic acid), 3.25 (dd, $1 \mathrm{H}, \mathrm{H}-3$ of oleanolic acid), 3.55-3.58 $(\mathrm{m}, 1 \mathrm{H}$, $\left.\mathrm{CH}_{2} \mathrm{OH}\right), 3.81-3.84\left(\mathrm{~m}, 1 \mathrm{H}, \mathrm{CH}_{2} \mathrm{OH}\right), 4.08\left(\mathrm{~m}, 2 \mathrm{H}, \mathrm{CH}_{2} \mathrm{OC}=\mathrm{O}\right), 4.20$ $\left(\mathrm{m}, 1 \mathrm{H}, \mathrm{H}-5^{\mathrm{Rha}}\right), 4.31-4.33\left(\mathrm{~m}, 1 \mathrm{H}, \mathrm{H}-5^{\mathrm{Rha}}\right), 5.03(\mathrm{~d}, 1 \mathrm{H}, J 2.4 \mathrm{~Hz}$, $\mathrm{H}-1^{\text {Rha }}$ ), 5.09 (d, $\left.1 \mathrm{H}, \mathrm{J} 2.1 \mathrm{~Hz}, \mathrm{H}-1^{\text {Rha }}\right), 5.32$ (br s, $1 \mathrm{H}, \mathrm{H}-12$ of oleanolic acid), 5.66-5.88 (m, 6H), 7.28-8.14 (m, 30H, 6Ph). ${ }^{13} \mathrm{C}$ NMR $\left(100 \mathrm{MHz}, \mathrm{CDCl}_{3}\right): 178.4,166.5,166.3,166.2$ (2C), 166.1, 166.0, 144.5, 134.0 (2C), 133.9, 133.7 (2C), 130.5, 130.3 (2C), 130.2, 130.1, 130.0, 129.9, 129.8 (2C), 129.4, 129.2, 129.0, 128.9, 124.5, 100.3 (C-1), 98.2 (C-1), 90.0, 73.4, 72.5 (2C), 71.8, 71.6, 70.8, 70.7, 68.9, 67.4, 67.2, 64.8, 56.0, 48.2, 47.3, 46.5, 42.3, 41.9, 40.0, 39.6, $39.0,37.4,34.5,33.7,33.3,33.1,31.3,30.0,29.2,29.0,28.2,26.6$, 26.4, 26.0, 24.2, 24.1, 23.6, 19.8, 18.9, 18.3, 18.1, 17.7, 17.2, 16.0. Anal. Calcd for $\mathrm{C}_{90} \mathrm{H}_{104} \mathrm{O}_{18}$ : C, 73.35; H, 7.11; Found: $\mathrm{C}, 73.06 ; \mathrm{H}$, 7.18 .

3.11. 3-O-[ $\alpha$-L-Rhamnopyranosyl]oleanolic acid $28-0$-[6-O-( $\alpha$-Lrhamnopyrano syl)hexyl] ester (6)

To a solution of 19 (148 mg, $0.1 \mathrm{mmol}$ ) in dry $\mathrm{CH}_{2} \mathrm{Cl}_{2} / \mathrm{MeOH}$ $(1: 2, \mathrm{v} / \mathrm{v}, 9 \mathrm{~mL})$ was added $1 \mathrm{M} \mathrm{NaOMe}(12 \mu \mathrm{L}, 0.012 \mathrm{mmol})$ in $\mathrm{MeOH}$. The mixture was stirred at rt until all starting materials were consumed, and then neutralized with Dowex-50 $\left(\mathrm{H}^{+}\right)$ion exchange resin. Filtration, concentration of the filtrate, and purification of the resulting residue by $\mathrm{LH}-20$ column chromatography using $\mathrm{H}_{2} \mathrm{O}$ as eluent afforded compound $6(76 \mathrm{mg}, 89 \%)$ as a white powder: $\left[\alpha_{\mathrm{D}}^{25}+6\right.$ (c 1.2 , water); ${ }^{1} \mathrm{H}$ NMR $(400 \mathrm{MHz}$, MeOD): $0.77,0.80,0.92,0.95,0.96,0.97,1.18(7 \mathrm{~s}, 7 \times 3 \mathrm{H}$, $\left.7 \mathrm{CH}_{3}\right), 1.24,1.27\left(2 \mathrm{~d}, 2 \times 3 \mathrm{H}, J 6.3 \mathrm{~Hz}, \mathrm{H}-6^{\mathrm{Rha}}\right), 0.77-2.02(\mathrm{~m}$, $30 \mathrm{H}), 2.91$ (dd, $1 \mathrm{H}, \mathrm{H}-18$ of oleanolic acid), 3.10 (dd, $1 \mathrm{H}, J$ 4.6, $11.5 \mathrm{~Hz}, \mathrm{H}-3$ of oleanolic acid), 3.31-3.41 (m, 3H), 3.56-3.72 $(\mathrm{m}, 5 \mathrm{H}), 3.79-3.82(\mathrm{~m}, 1 \mathrm{H}), 3.83-3.85(\mathrm{~m}, 1 \mathrm{H}), 4.03(\mathrm{t}, 2 \mathrm{H}, J$ $6.2 \mathrm{~Hz}), 4.66\left(\mathrm{~d}, 1 \mathrm{H}, J 1.6 \mathrm{~Hz}, \mathrm{H}-1^{\mathrm{Rha}}\right), 4.74(\mathrm{~d}, 1 \mathrm{H}, J 1.4 \mathrm{~Hz}$, $\mathrm{H}-1^{\text {Rha }}$ ), 5.27 (br s, $1 \mathrm{H}, \mathrm{H}-12$ of oleanolic acid). ${ }^{13} \mathrm{C}$ NMR (100 MHz, MeOD): 178.7, 144.3, 123.0, 103.6 (C-1), 100.8 (C-1), $89.5,73.3,73.2,71.7,71.5,69.1,68.9,67.6,64.7,56.8,47.6$, $42.1,42.0,39.9,39.2,38.8,37.1,34.0,33.1,32.9,32.7,30.8$, $29.9,28.9,28.0,26.3,26.1,25.8,25.6,23.8,23.2,18.6,17.3$, 17.1, 17.0, 16.2, 15.2. MALDITOF-MS: calcd for $\mathrm{C}_{48} \mathrm{H}_{80} \mathrm{O}_{12}$ : $848.56[\mathrm{M}]^{+}$; found, $871.72[\mathrm{M}+\mathrm{Na}]^{+}$. Anal. Calcd for $\mathrm{C}_{48} \mathrm{H}_{80} \mathrm{O}_{12}$ : C, 67.89; H, 9.50; Found: C, 67.63; H, 9.44.

\subsection{Cell culture assay}

HL-60 cells were maintained in the RPMI 1640 medium containing $10 \%$ fetal bovin serum supplemented with L-glutamine, 100 units/mL penicillin, and $100 \mu \mathrm{g} / \mathrm{mL}$ streptomycin. The leukemia cells were washed and suspended in the above medium to $1 \times 10^{5}$ cells $/ \mathrm{mL}$, and $100 \mu \mathrm{L}$ of this cell suspension was placed in each well of a 96-well plate. The cells were incubated in $5 \% \mathrm{CO}_{2}$ /air for $24 \mathrm{~h}$ at $37^{\circ} \mathrm{C}$. After incubation, $3 \mu \mathrm{L} / \mathrm{mL}$ of DMSO- $\mathrm{H}_{2} \mathrm{O}(1: 20, \mathrm{v} / \mathrm{v})$ solution containing the sample was added to give the final concentration of $0.1-30 \mu \mathrm{L} / \mathrm{mL}$, while $3 \mu \mathrm{L} / \mathrm{mL}$ of $\mathrm{DMSO}-\mathrm{H}_{2} \mathrm{O}(1: 20, \mathrm{v} / \mathrm{v})$ was added into control wells. The cells were further incubated for $72 \mathrm{~h}$ in the presence of each agent, and then cell growth was evaluated using modified 3-(4,5-dimethylthiazol-2-yl)-2,5-diphenyltetrazolium bromide (MTT) reduction assay, and the $\mathrm{IC}_{50}$ values were calculated accordingly. ${ }^{31,32}$ 


\section{Acknowledgment}

This work was supported in partial by NNSF of China (Projects 20621703, 20872172, and 20732001).

\section{Supplementary data}

Supplementary data associated with this article can be found, in the online version, at doi:10.1016/j.carres.2009.04.005.

\section{References}

1. Kalinin, V. I.; Silchenko, A. S.; Avilov, S. A.; Stonik, V. A.; Smirnov, A. V. Phytochem. Rev. 2005, 4, 221-236.

2. Kalinowska, M.; Zimowski, J.; Paczkowski, C.; Wojciechowski, Z. A. Phytochem. Rev. 2005, 4, 237-257.

3. Park, J. D.; Rhee, D. K.; Lee, Y. H. Phytochem. Rev. 2005, 4, 159-175.

4. Lanzotti, V. Phytochem. Rev. 2005, 4, 95-110.

5. Waller, G. R.; Yamasaki, K. Saponins Used in Traditional and Modern Medicine: Plenum Press: New York, 1996. and references cited therein.

6. Yu, B.; Zhang, Y.; Tang, P. Eur. J. Org. Chem. 2007, 5145-5161.

7. Hostettmann, K.; Marston, A. Saponins; Cambridge University Press: Cambridge, UK, 1995.

8. Cheng, S.; Du, Y.; Bing, F.; Zhang, G. Carbohydr. Res. 2008, 343, 462-469.

9. Bing, F. H.; Zhang, G. B. Hubei J. Tradit. Chin. Med. 2005, 27, 48-49.

10. Bing, F. H.; Yi, Y. D.; Zhang, G. B. J. Chin. Pharm. Univ. 2005, 36, 338-341.

11. Zhao, L.; Chen, W. M.; Fang, Q. C. Planta Med. 1991, 57, 572-574.
12. Chang, L. C.; Tsai, T. R.; Wang, J. J.; Lin, C. N.; Kuo, K. W. Biochem. Biophys. Res. Commun. 1998, 242, 21-25.

13. Jeong, H. G.; Choi, C. Y. Planta Med. 2002, 68, 392-396.

14. Barthomeuf, C.; Boivin, D. Cancer Chemother. Pharmacol. 2004, 54, 432-440.

15. Debiton, E.; Borel, M.; Communal, Y.; Mshvildadze, V.; Barthomeuf, C. Melanoma Res. 2004, 14, 97-105.

16. Yu, B.; Xie, J.; Deng, S.; Hui, Y. J. Am. Chem. Soc. 1999, 121, 12196-12197.

17. Randolph, J. T.; Danishefsky, S. J. J. Am. Chem. Soc. 1995, 117, 5693-5700.

18. Nishizawa, M.; Yamada, H. Synlett 1995, 785-793.

19. Deng, S.; Yu, B.; Hui, Y. Tetrahedron Lett. 1998, 39, 6511-6514.

20. Deng, S.; Yu, B.; Lou, Y.; Hui, Y. J. Org. Chem. 1999, 64, 202-208.

21. Shi, B.; Tang, P.; Hu, X.; Liu, J.; Yu, B. J. Org. Chem. 2005, 70, 10354-10367.

22. Du, Y.; Gu, G.; Wei, G.; Hua, Y.; Linhardt, R. J. Org. Lett. 2003, 5, 3627-3630.

23. Gu, G.; Du, Y.; Linhardt, R. J. J. Org. Chem. 2004, 69, 5497-5500.

24. Zhang, M.; Du, Y.; Kong, F. Carbohydr. Res. 2001, 330, 319-324.

25. Osborn, H. M. I.; Brome, V. A.; Harwood, L. M.; Suthers, W. G. Carbohydr. Res. 2001, 332, 157-166

26. Zhang, Z.; Magnusson, G. Carbohydr. Res. 1996, 295, 41-55.

27. Du, Y.; Pang, Q.; Kong, F. Carbohydr. Res. 2000, 329, 17-24.

28. Schmidt, R. R.; Toepfer, A. Tetrahedron Lett. 1991, 32, 3353-3356.

29. Yan, M. C.; Liu, Y.; Chen, H.; Ke, Y.; Xu, Q. C.; Cheng, M. S. Bioorg. Med. Chem. Lett. 2006, 16, 4200-4204.

30. Zhang, Y. N.; Zhang, W.; Hong, D.; Shi, L.; Shen, Q.; Li, J. Y.; Li, J.; Hu, L. H. Bioorg Med. Chem. 2008, 16, 8697-8705.

31. Mosmann, T. J. Immunol. Methods 1983, 65, 55-61.

32. Sargent, J. M.; Taylor, C. G. Br. J. Cancer 1989, 60, 206-210.

33. Melek, F. R.; Miyase, T.; Ghaly, N. S. Phytochemistry 2003, 62, 557-562.

34. Yokosuka, A.; Kawakami, S.; Haraguchi, M.; Mimaki, Y. Tetrahedron 2008, 64 1474-1481. 